\title{
Task Allocation in Hybrid Big Data Analytics for Urban loT Applications
}

WEILONG DING and ZHUOFENG ZHAO, School of Information Science and Technology, North China University of Technology, Beijing, China and Beijing Key Laboratory on Integration and Analysis of Large-scale Stream Data, Beijing, China

JIANWU WANG, Department of Information Systems, University of Maryland, Baltimore, MD, U.S.A.

HAN LI, School of Information Science and Technology, North China University of Technology, Beijing, China and Beijing Key Laboratory on Integration and Analysis of Large-scale Stream Data, Beijing, China

In urban Internet of Things (IoT) environments, data generated in real time could be processed by analytical applications in online or offline mode. In the management perspective of runtime environments, such modes can hardly be supported in a unified framework under multiple restrictions such as latency, utility, and QoS (quality of service). Meanwhile in the optimization perspective of specific applications, it is difficult for current infrastructure to efficiently allocate sufficient resources to tasks of an application, simultaneously considering multiple factors such as data size, velocity, and locality. In this article, two task allocation methods are proposed for batch and stream analytics to improve resource utility with auto-scaling guarantee when an analytical application is submitted or sudden workloads appear. Taking the highway domain as an example, the task allocation methods are implemented in a novel combined framework accordingly. Using both real-world and simulated data, extensive experiments show that our methods can improve utility efficiency with effective offload support.

CCS Concepts: $\bullet$ Information systems $\rightarrow$ Stream management; Online analytical processing engines; Computing platforms; Spatial-temporal systems;

Additional Key Words and Phrases: Internet of Things, analytical framework, task allocation, urban computing, spatio-temporal data

ACM Reference format:

Weilong Ding, Zhuofeng Zhao, Jianwu Wang, and Han Li. 2020. Task Allocation in Hybrid Big Data Analytics for Urban IoT Applications. ACM/IMS Trans. Data Sci. 1, 3, Article 18 (September 2020), 22 pages.

https://doi.org/10.1145/3374751

This work was supported by National Key R\&D Program of China (No. 2018YFB1402500), National Natural Science Foundation of China (No. 61702014), Beijing Municipal Natural Science Foundation (No. 4192020, No. 4202021), and the Top Young Innovative Talents of North China University of Technology (No. XN018022).

Corresponding authors' addresses: No. 5 Jinyuanzhuang Road, Shijingshan District, North China University of Technology, Beijing, 100144, China.

Authors' addresses: W. Ding (corresponding author), Z. Zhao, and H. Li, 805 Hanxue Building, School of Information Science and Technology, North China University of Technology, No. 5 Jinyuanzhuang Road, Shijingshan District, Beijing, China; emails: \{dingweilong, edzhao, lihan\}@ncut.edu.cn; J. Wang, ITE 423, Department of Information Systems, University of Maryland, Baltimore County, Baltimore, MD, U.S.A.; email: jianwu@umbc.edu.

Permission to make digital or hard copies of all or part of this work for personal or classroom use is granted without fee provided that copies are not made or distributed for profit or commercial advantage and that copies bear this notice and the full citation on the first page. Copyrights for components of this work owned by others than ACM must be honored. Abstracting with credit is permitted. To copy otherwise, or republish, to post on servers or to redistribute to lists, requires prior specific permission and/or a fee. Request permissions from permissions@acm.org.

(C) 2020 Association for Computing Machinery.

2577-3224/2020/09-ART18 \$15.00

https://doi.org/10.1145/3374751

ACM/IMS Transactions on Data Science, Vol. 1, No. 3, Article 18. Publication date: September 2020. 


\section{INTRODUCTION}

With extensively deployed sensors in modern cities, Big Data have been widely adopted for domain analyses in recent years to build urban Internet of Things (IoT). For instance, there are surveillance data from cameras on trunk roads, smart-card data from readers in buses, statistical data from inductive loops at toll stations, and status data from transducer in power plants. From highly distributed IoT devices, such sensory data often require real-time and latency-sensitive analytics [Mahmud et al. 2018]. It could be used to comprehend current urban situation and predict future trends in different views. For example, in the inter-city transportation domain, the toll data from toll stations are used to evaluate highway traffic every 5 minutes [Ding and Zhao 2018]; in the intra-city transportation domain, surveillance data from cameras on roads can be employed to detect illegal vehicles and their abnormal behaviors [Ding et al. 2016]; in the power grid domain, the smart meter data from multiple sensors could be aggregated for urban electricity rationing [Nazmudeen et al. 2016].

Two characteristics appear in these analytical applications on IoT sensory data. On the one hand, most of such IoT data are captured as spatio-temporal data streams [Perera et al. 2017], and the applications often perform as online analytics. A toll station in Henan province, China, generates a toll record whenever a vehicle is entering or exiting. About every 7 minutes, smart meters in the State Grid of China send the upper datacenter a record including accumulative electricity consumption and recent device states. All those types of data are spatio-temporal with attributes of locations and times in a real-time manner. The long-term data are prone to store in the Cloud permanently, but some short-term ones are in one-off usage through stream analytics. On the other hand, analytical applications on such data are traditionally executed in a centralized backend where most end users can access results [Yu et al. 2018]. For example, in a private Cloud, multi-perspective highway traffic statistics [Ding et al. 2020] are performed on massive toll data for business technicians. However, a centralized Cloud as a physical far-end may make the response latency of the applications unacceptable when urban sensors grow to huge numbers with complex structure. To reduce the latency, it is a trend nowadays to offload some computational tasks of applications to edge servers [Ferrer et al. 2019] through prosperous Edge Computing techniques [Lopez et al. 2015]. In general, the term "Edge"1 depicts all the possible computing resources where data pass through from front-end sensors to far-end Cloud.

However, Big Data analytics for urban IoT applications still remain challenging to hold their favorable quality of service (QoS), especially under dynamic workloads. First, from a management perspective of the runtime environment, it is difficult to support both batch and stream computational tasks of multiple analytical applications in one framework under diverse restrictions. Besides sensitive latency during data processing and transmission, accuracy is also the focus in business statistics. Although current stream computing frameworks are powerful, they cannot completely replace general batch computing ones [Locher and Sima 2016] due to the precision requirement of offline massive data analytics. Accordingly, it is common to employ independent frameworks in shared business resources and imperative to improve overall utilities for multiple goals in one runtime environment. Taking the highway domain as an example, online short-term prediction of traffic volumes is required for current situation evaluation, and offline long-term prediction is valuable for policy guidance on coming holidays. If those applications are executed in different native systems (e.g., Hadoop and Storm), then it brings more technical complexity for infrastructure to manage diverse computing models [Ding et al. 2020]. Second, from the optimization perspective of specific data analysis, it is difficult to efficiently allocate

\footnotetext{
${ }^{1}$ Although there are slight differences and open controversy in specific perspectives, "Edge Computing" and "Fog Computing" are regarded as synonyms in this article according to the popular standpoint in recent works.
} 
sufficient resources to tasks for an application, considering multiple factors like data size, velocity, and locality simultaneously. Elastic auto-scaling is required for the runtime environment when analytical applications are submitted or dynamic workloads appear. Current popular frameworks like Hadoop, Storm, or Spark do not support dynamic configuration without intervention to match long-running workload [Mach and Becvar 2017]. When load bursts, the QoS of applications would jitter at times; while load is low, redundant resources are wasted. Taking the power grid domain as example, the sensory data of 25 million smart meters in the Chinese city Hangzhou would be uploaded at 00:00:00 every day. Such a bursting load in a very short period always brings several hours' delay for analytical applications, especially in some big residential communities due to improper resource allocation under workload imbalance.

To deal with the above problems, two task allocation methods are presented to improve resource utilization in one runtime environment. Our contributions can be concluded as follows. (1) The batch and stream computing models are supported collaboratively in a unified framework, where Cloud and Edge resources are effectively supplied for urban IoT applications. With a reasonable execution mechanism, diverse data analytical requirements can be satisfied within the unified framework. (2) Our methods achieve quasi-optimal resource utilization with auto-scaling guaranteed in two main conditions with available Cloud-Edge resources. When an analytical application is initially submitted, the task placement method through particle swarm optimization is to reduce the consumption of time and communication in a workload-agnostic way. When bursting (shrinking) workload appears, the adaptive task scheduling through workload prediction can find straggler tasks (spare tasks) and migrate them proactively to satisfy QoS continuously in a workload-aware way. (3) Our work shows convincing utilization benefits for the runtime environment on both real and simulated data in extensive experiments.

The rest of this article is organized as follows. Section 2 discusses related works in three perspectives. Section 3 presents preliminaries including motivation and methodology. Two task allocation methods are elaborated in Section 4. Section 5 quantitatively evaluates the effects in extensive experiments. We summarize conclusion in Section 6.

\section{RELATED WORK}

\subsection{Hybrid Analytical Paradigm on Batch and Stream Data}

On urban IoT data, stream computing model is necessary for analytical applications. As a memorybased processing without storing data on disk first, such an online model may not always guarantee high accuracy due to the limited data amount and lack of locality awareness [Salmon et al. 2015]. Therefore, many analytical applications require the processing on both batch and stream data. It has become a hot topic since 2014 on how to achieve a hybrid analytical paradigm on such data.

Four types of hybrid analytical paradigms exist based on their involved technologies. The first is unified data abstraction. With the stream abstraction Discretized Streams (D-Streams) over Resilient Distributed Datasets (RDDs) data structure [Zaharia 2014], both batch and stream computing models can be supported in the Spark eco-system. Due to its micro-batch implementation, the processing latency of Spark Streaming is worse than sub-second level. In the intra-city transportation domain, a hybrid solution is proposed [Zhao et al. 2015] to support offline and online analytics on the traffic sensory data. To detect illegal vehicles in multiple patterns, dedicated data abstraction, spatio-temporal index, and storage policies are employed, but they are hardly extended and applied in other domains. The second is a batch-based compatible model to support stream analytics. Based on the standard batch model of Hadoop MapReduce, the pipelinebased incremental update [Cao and Wang 2015] and tuple-level latency restriction [Li 2015] are extended respectively to build and schedule tasks of stream computing. But the processing latency 
of such models still has a lower bound of sub-second due to inherent limitation of batch model. The third is the stream-based compatible model to support batch analytics. Based on Storm, Pig Squeal [Lampton 2015] adds incrementally an updated batch, and Cyclone [Locher and Sima 2016] translates codes to run Hadoop MapReduce jobs in a stream processing system. The technical complexity of these two technologies is too high to be applied in some critical domains. The fourth is called a federal-based combination. Middleware assigns applications with their tasks to respective native batch or stream systems. Facebook provides a real-time analytical solution [Chen et al. 2016] to combine its own components Puma with native Hadoop. Another general architecture for such a combination is proposed [Zhu et al. 2016] with the layers of storage, data processing, analytics, and decision-making, which inspires the work of this article. Some other tools are used to define abstract applications on such a combination. Twitter Summingbird imports dedicated language for a developer to transparently integrate Hadoop and Storm [Boykin et al. 2014]. Analogously, a unified interface, called Apache Beam [Carbone et al. 2015], completes batch and streaming processing in independent Spark or Flink. All such combination solutions are very compatible with existing native systems in a low intrusive way but require complex guarantee for task allocation, load balance, and high availability. In summary, four types of hybrid analytical paradigms have their own characteristics with limitations and imply the tradeoff between programming complexity and management flexibility.

Our work lies in the fourth paradigm with the advantage of low-intrusion and highcompatibility. Some related frameworks have been presented in specific domains, such as a combined platform [Zhang et al. 2017] in petrolic data analytics, a collaborative computing [Ding et al. 2017] in urban transportation, and a platform service [Ding et al. 2020] in highway. However, the major challenges for task assignment and resource management [Cho et al. 2016] have not been fully addressed in urban IoT environment yet.

\subsection{Task Allocation in the Cloud-Edge Environment}

In IoT environments, edges are regarded as any computing and network resources along the path between data sources and cloud data centers [Shi et al. 2016], like routers, sensors, and network edge servers. In recent years, such inexpensive resources are considered to achieve fast response on dynamic workload. Accordingly, offloading techniques have been studied to allocate applications' tasks to right locations for execution. It can reduce traffic and computational pressure in some bottleneck nodes. The offloading in IoT can be divided into three categories based on their target locations [Premsankar et al. 2018]: resource-rich servers near devices, heterogeneous edge nodes, and Edge-Cloud collaboration. Each of them implies different tradeoff between computing latency and transmission consumption, and the most suitable applications for offloading would require lots of computational resources with small data in communications [Mach and Becvar 2017].

Our work in this article belongs to the last category, considering the advantage of high resource utilization. A collaboration of edges and public Cloud is proposed [Farris et al. 2015] for IoT applications, where edge nodes can be orchestrated dynamically to maximize the number of runnable tasks. For real-time processing on geographical edge nodes, offloading is employed to support low latency and mobile applications in the middle layer [Mukherjee et al. 2018]. Additionally, proper parameters play an important role in performance before offloading. A dynamical configuration learning algorithm [Jayaraman et al. 2015] is designed to predict applications' performance to enable fine-grained offloading control. However, none of them fully address the application offloading for hybrid models of stream and batch computing.

Among distributed edge nodes, computing offloading is traditionally carried out by the migration of Virtual Machines (VM). To reduce infrastructure cost and migration latency, different lightweight solutions have been studied. In works Ismail et al. [2015] and Pahl et al. [2016], 
Docker-container-based edge collaboration is proposed and qualitatively evaluated. Such solutions show efficient interoperability in application registry [Saurez et al. 2016], lifecycle government [Yangui et al. 2016], and live tasks migration [Machen et al. 2016] in IoT.

In this article, we borrow the idea of computing offloading to design a hierarchical framework for Cloud-Edge resource collaboration. It guarantees low latency during allocating tasks of urban IoT analytical applications due to fast response of edges.

\subsection{Task Allocation for Distributed Real-time Data Analytics}

Data analytics in large companies or systems always spans to multiple servers, and parallel processing is often difficult in window- or sequence-based tasks [Sattler and Beier 2013]. It poses challenges for real-time data analytics to achieve elasticity and efficient decisions by instant workload. Most of available data-processing frameworks, such as Spark and Storm, do not provide enough flexibility, because their architectures are based on static configurations and do not allow dynamic adjustment. The "elasticity" of stream computing refers to the ability in system to allocate additional resources or release idle ones to match the workload [Dias de Assunção et al. 2018]. Existing approaches to achieve such elasticity can be categorized into two types. The first type does static task allocation before job execution. Like the query plan optimization in a database, some studies [Peng et al. 2015; Tolosana-Calasanz et al. 2016] reconfigure the runtime physical plan of a job according to its build-time logical plan (e.g., topology in Storm) to reduce data communication among tasks of different nodes. The second type does online dynamic task allocation during job execution proactively or reactively. It comprises both organization of available resource and dynamic allocation of tasks for better overall utilization. For specific bottleneck tasks whose capacity cannot keep up with the rate of incoming tuples, a scaling policy is brought to determine when scaling out/up plans should be performed [Lohrmann et al. 2015]. To optimize resource utilization, such reactive strategy introduces two operations, namely Rebalance and ResolveBottlenecks, to adjust the parallelism of tasks and resolve bottlenecks, respectively. Analogously, two customized Storm schedulers are proposed [Aniello et al. 2013] for respective offline static task assignment and for dynamic scheduling to reduce the communication of inter-process or inter-node. In the Microsoft framework TimeStream, a resilient substitution is advocated to handle failure and reconfiguration due to dynamic workload [Qian et al. 2013]. With the declarative programming model, it shows robust scalability with low latency guarantee. In Google MillWheel, a combination of scalability, fault tolerance, and a versatile programming model is proposed [Akidau et al. 2013] to implement elastic stream computing. In addition, some frameworks, like Apache Edgent, ${ }^{2}$ focus on real-time data analytics in heterogeneous edge nodes. However, most of the existing works remain at an architectural level and would only execute in Cloud. The task allocation still faces challenges in IoT environment to guarantee QoS properties such as low latency.

As long-running stream analytics cannot be restarted without data loss, its initial task allocation (i.e., task placement) on available resources is more critical [Dias de Assunção et al. 2018]. For example, a simple greedy task placement mechanism can be used to pursue stable performance [Ding et al. 2016]. Multiple algorithms and models have been applied to achieve better QoS (e.g., lower completion time, smaller transmitted data, fewer resource cost, and less privacy risk.) in a decentralized environment [Masdari et al. 2017]. However, most of them depend on only Cloud resource without edges [Masdari et al. 2016]. In this article, two task allocation methods are presented in respective phases with edge support to improve QoS and resource utilization.

\footnotetext{
$\overline{{ }^{2} \text { http://edgent.apache.org/. }}$
} 
Table 1. The Structure of Toll Data

\begin{tabular}{lll}
\hline Attribute & \multicolumn{1}{c}{ Notation } & Type \\
\hline collector_id & toll collector identity & Entity \\
vehicle_license & vehicle identity & Entity \\
vehicle_type & vehicle type & Entity \\
card_id & vehicle passing card identity & Entity \\
etc_id & vehicle ETC card identity & Entity \\
etc_cpu_id & ETC card chip identity & Entity \\
entry_time & vehicle entry timestamp & Time \\
exit_time & vehicle exit timestamp & Time \\
entry_station & identity of entry station & Space \\
entry_lane & lane number of entry station & Space \\
exit_station & identity of exit station & Space \\
exit_lane & lane number of exit station & Space \\
\hline
\end{tabular}

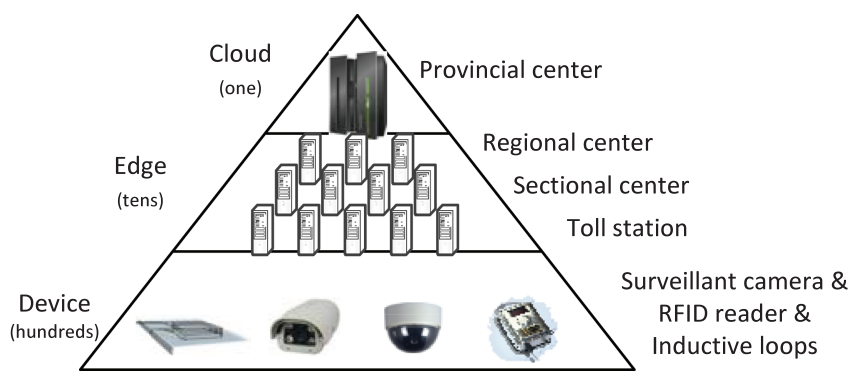

Fig. 1. IoT deployment in highway domain.

\section{PRELIMINARIES}

\subsection{Motivation}

Our research originates from the Highway Big Data Analysis System of Henan, which is the most populated province in China. The system we built has been in production since October 2017 and is expected to improve routine business analytics for highway management through Big Data technologies. Operated by officers of Henan Transport Department, billions of records in recent 2 years have been stored in the system, involving 660 million vehicles, 37 highway lines, and 343 toll stations. Besides such historical data, multiple types of continuous ones, such as toll data, meteorology data, and automatic license plate data, are channelled into the system. Taking the toll data in Table 1 as an example, each record contains 12 attributes that include 6 entities, 2 temporal attributes, and 4 spatial ones. It is typical spatio-temporal data.

Toll data are generated continuously from surveillance cameras with the functions of image recognition and RFID readers, pass through hierarchical edges, and are eventually sent to a private Cloud at provincial data center. As a typical design in highway domain, edges are the servers at a sectional center and a regional center connected by a private network, which is constructed as a tree topology as Figure 1. An edge server in a given level only uploads data to one edge server in its upper level and gathers data from multiple edge servers in its lower level. Compared with a mobile edge computing environment, edge servers here are more powerful commercial machines with certain computing capacity and data storage. 


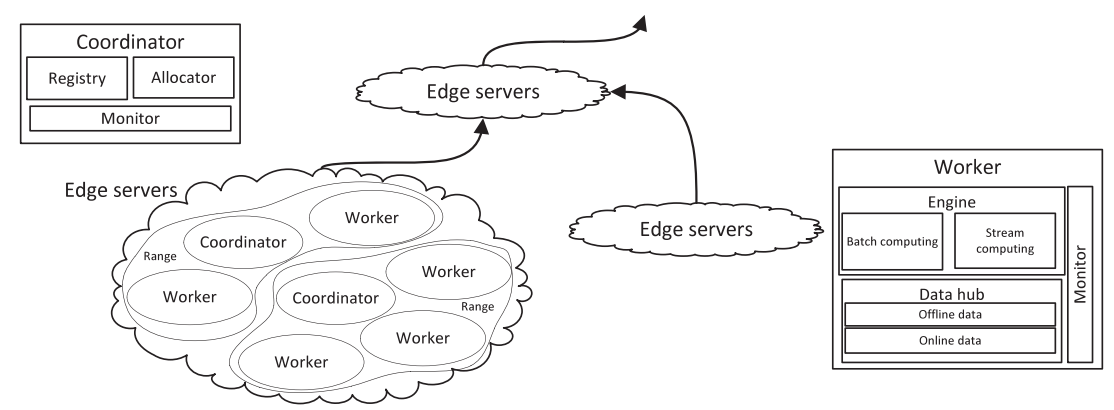

Fig. 2. Architecture and components of the framework.

Our developed system supports multiple data analytical applications. For example, the traffic volume application counts the vehicles passing a spatial location in given time slots not only for current statistics estimation but also for future traffic guidance [Lv et al. 2015]. Some problems still remain for such analytics without appropriate remedies in practice. On the one hand, real-time estimation requires a low latency guarantee, and long-term trend prediction demands short makespan with high precision. Therefore, analytical applications of online stream and offline batch run in different native frameworks like Storm and Hadoop. For example, the online traffic volume statistics and its prediction are triggered to run every 5 minutes; the offline monthly traffic volume of ETC vehicles and monthly proportion of vehicle type would be executed once a month. The progressive increase of these applications makes business management difficult and inefficient. On the other hand, QoS guarantee is not trivial for a specific application due to complex instant workload. During workday rush hours, two workload peaks appear at most of the toll stations due to busy traffic. On some holidays, like Labor Day and National Day, the bursting workloads in a whole day would deteriorate many QoS of analytical applications. To achieve lower response time or shorter completion time, we should consider how to appropriately allocate tasks of critical applications to adequate resources, especially to edges. Instead of running only in the central Cloud, some tasks would perform faster if they are on edge servers close to the data.

Therefore, it is necessary in this specific domain to enhance data analytics with hybrid data flexibly and efficiently. Such interesting observations are the very motivation of this article.

\subsection{Architecture and Methodology}

As shown in Figure 2, our proposed framework has two types of components: coordinator and worker. Each component is on an edge or Cloud logical server that would be a physical machine, a virtual machine, or a Linux container (e.g. Docker). We assume that the edges compose treelike hierarchy like Figure 1. In many domains like highway, power grid, and urban transportation, it is sound because obvious geographic dispersion of edge servers is in levels, and the levels are orthogonal.

(1) Each coordinator encapsulates master nodes of native data processing systems. It maintains the metadata, monitors the status of given components, and allocates tasks of analytical applications. Three modules are included: registry, monitor, and allocator. The registry stores metadata of workers in the same range and the analytical applications on those workers. Supervising those workers every $5 \mathrm{~s}$, the coordinator monitor gathers status, probes resource utilization, and evaluates each application's QoS. The allocator is a dedicated unit to dispatch tasks when an application is submitted or sudden workload appears. Through Remote Procedure Call (RPC) interfaces with masters of native systems, 
task allocation methods can be plugged in here. By extending AbstractYarnScheduler for offline batch analytics in Hadoop MapReduce or implementing IScheduler for online stream analytics in Storm, user-defined allocation methods can be assembled and triggered to execute in allocator.

(2) Each worker is a computing node and provides a runtime environment for analytical applications. Three modules are in a worker: data hub, monitor, and engine. The data hub maintains local data: Online continuous data are acquired from other workers through message broker, and offline historical data are managed by a lightweight relational database, distributed file system, or NoSQL storage. The worker monitor would probe resource utilization, each task's QoS, and workload every second. The metadata of those modules would be updated to the registry of coordinator periodically. The engine is a middleware to combine native batch and stream computing systems. For example, Hadoop and Storm can be combined federally by encapsulation here where required data can be achieved from data hub.

(3) Among some servers of the same edge level (e.g., toll station, sectional center, or regional center of highway domain) in hierarchy, a coordinator and the workers under its surveillance compose a range. Range is actually an independent native cluster system (e.g., Hadoop, Spark, or Storm) to which analytical applications can be submitted. For high availability, multiple coordinators in the same range run as active standby, and only one takes charges actively. A coordinator would broadcast the update of metadata (e.g., local data variance of workers) to another one in its upper level and can allocate tasks to the worker in the same range or to a coordinator in its lower levels for further dispatching.

Considering the compatibility with existing batch or stream computing jobs in a low intrusive way, a dedicated configuration is defined for an analytical application.

Definition 1 (Application). An analytical application is defined as a capsulation of one computing job: application::=<profile, jobType, offloadOpt, input, qos, output $>$. The profile is the basic information of the application, including its name, owner, and creator. The jobType:: = batch|stream presents the underlying computing model. The offloadOpt:: = true|false indicates whether the application would be offloaded to edges in lower levels. If it is true, then offloadLevel:: $=[$ all, $1 . . n]$ implies the offload employs the servers at most the $i$ th lower level, and $i \in 1 . . n$; offloadNum:: $=[$ all, $1 . . \mathrm{m}] \mathrm{im}-$ plies that the offload involves at most $j$ servers of each level, $j \in 1 . . m . m$ and $n$ is a positive integer respectively. The input:: $=<$ jobPackage, otherParameters $>$ are input parameters including the location of code package. The qos::= [latency, cpu, memory, bandwidth] shows user-defined QoS for the application. The output:: $=<$ structuralParameters $>$ is output parameters as structural JSON object.

We illustrate how to use the definition above in the scenario of Section 3.1. Table 2 shows an application to count the traffic volume at stations by packaging a batch computing job of Hadoop. Due to the semantic of statistical summary, lower edges can be used for the application offloading and gathering results independently. The referred Hadoop MapReduce job and its input parameters are assigned. Here, the job is initiated from a service template [Ding et al. 2020] with its multidimensional parameters. QoS requirements are also designated in detail. The output is a structural composition of identifier (i.e., toll station, section, or region) with its traffic volume.

Such application definition includes user-defined requirements for executive conditions like the backend framework and QoS. In our current version, "batch" applications are executed in Hadoop MapReduce, and "stream" ones are in Storm. It comes from the safety fact that a single framework for either computing model can avoid the complexity of interoperability [Kolajo et al. 2019]. By the allocator of coordinator, the application would be parsed as concrete tasks for allocation further. 
Table 2. The Definition of an Example Application

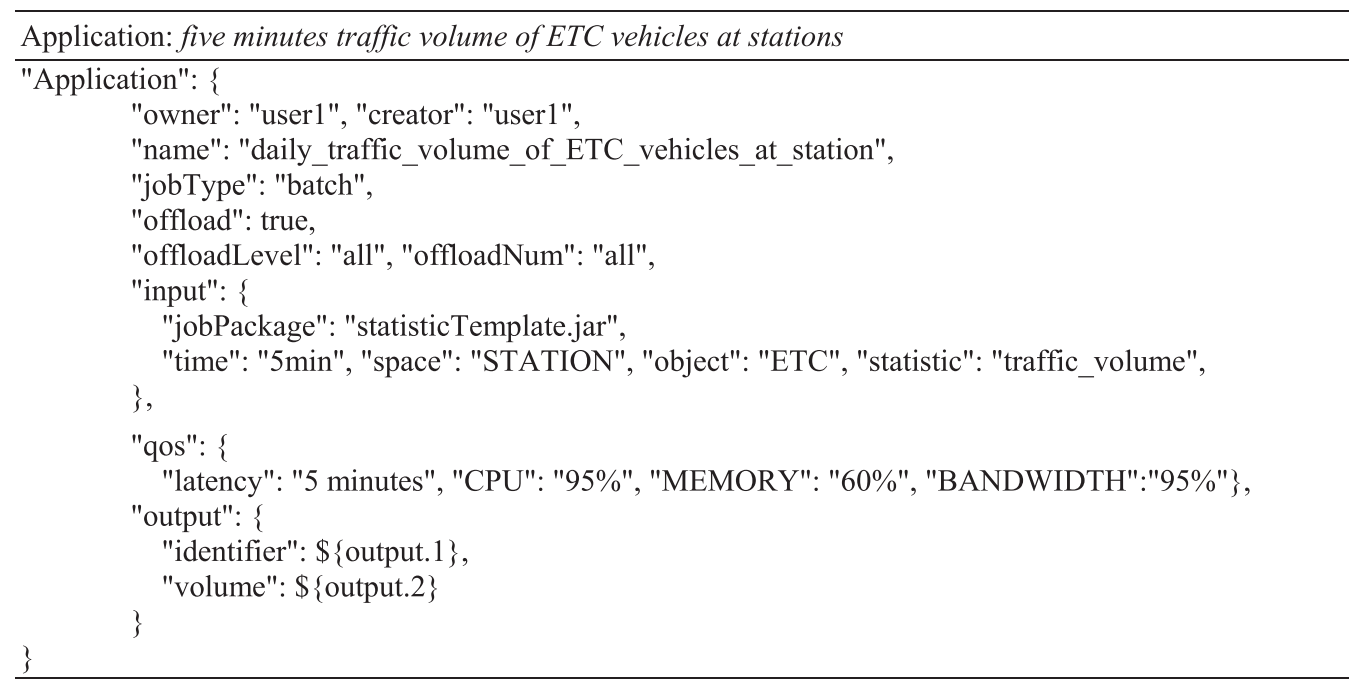

Note that although a specific domain is exemplified in this article, our work would benefit for other analogous ones like power grid and urban transportation where edges are in a tree hierarchy.

\section{TASK ALLOCATION METHODS IN TWO CONDITIONS}

In our framework, an application can be submitted to any coordinator in a range, and then its tasks would be allocated to involved workers. Two task allocation methods are proposed here in two conditions to improve resource utilization and QoS: one for initial task placement in a workload agnostic way and the other for sudden bursting or shrinking condition in a workloadaware fashion.

\subsection{Initial Task Placement for a Submitted Application}

After an application is submitted to a coordinator, its tasks would be dispatched to workers in the same range first and then to workers in other ranges of lower levels if necessary. The initial allocation for an application is termed as task placement in this article, which is a workload agnostic method based on Particle Swarm Optimization (PSO).

PSO is a powerful random technique to solve multimodal continuous optimization problems [Masdari et al. 2017]. A possible solution at time $t$ is presented as a particle $x_{i d}^{t}$ and would update in iterations based on Equation (1). Here, $v_{i d}^{t}$ is the velocity of this particle at time $t$. Based on previous velocity $\left(v_{i d}^{t}\right)$, inertia weight $\omega$, acceleration constants $c_{\mathrm{p}}$ and $c_{\mathrm{g}}$, and random numbers $r_{\mathrm{p}}$ and $r_{\mathrm{g}} \subset(0,1)$, the best position of the particle $p_{i d}^{t}$, and the best position of whole particles $p_{g d}^{t}$, the velocity of this particle in the next iteration $\left(v_{i d}^{t+1}\right)$ is determined by Equation (2). Then, as shown in Equation (3), the best position of particle $p_{i d}^{t+1}$ in the next iteration is found by fitness function $f$,

$$
\begin{aligned}
x_{i d}^{t+1} & =x_{i d}^{t}+v_{i d}^{t+1}, \\
v_{i d}^{t+1} & =\omega * v_{i d}^{t}+c_{\mathrm{p}} * r_{\mathrm{p}} *\left(p_{i d}^{t}-x_{i d}^{t}\right)+c_{\mathrm{g}} * r_{\mathrm{g}} *\left(p_{g d}^{t}-x_{i d}^{t}\right), \\
p_{i d}^{t+1} & =\left\{\begin{array}{cc}
x_{i d}^{t+1}, \quad \mathrm{f}\left(x_{i d}^{t+1}\right)<\mathrm{f}\left(p_{i d}^{t}\right) \\
p_{i d}^{t}, & \text { otherwise }
\end{array}\right.
\end{aligned}
$$

For the task placement in our framework, particle $x_{i d}^{t}$ should be encoded and decoded as follows. 
Definition 2 (Particle Encoding). A particle $x_{i d}^{t}=\left(x_{1}, x_{2}, \ldots, x_{i}, \ldots, x_{\mathrm{T}}\right)$ as a T-dimensional vector is a solution of task placement at the th iteration, and $\mathrm{T}$ is the task number of the application. The element $x_{i}=j$ implies the $i$ th task $t_{i}$ of an application would be placed on worker $r_{j}$, where $1 \leq$ integer $i \leq \mathrm{T}, 1 \leq$ integer $j \leq \mathrm{R}$, R is the number of workers.

Definition 3 (Particle Decoding). In the ith iteration, all the particles could be decoded as a resource utility matrix $Y_{l}$ whose element $Y_{l}(i, j)$ depicts resource usage of $l$ by task $t_{i}$ on worker $r_{j}$. Here, $1 \leq$ integer $i \leq \mathrm{T}, 1 \leq$ integer $j \leq \mathrm{R}$, resource $l=\mathrm{CPU}$, MEMORY or BANDWIDTH. Particularly, matrix $Y_{0}$ is termed as the placement on the worker nodes, whose element $Y_{0}(i, j)=0$ or 1 . If $Y_{0}(i, j)=1$, then task $t_{i}$ is placed on worker $r_{j}$.

To find the optimal solution, batch and stream analytical applications have to be considered respectively due to their distinct points of focus.

For a batch application, shorter completion time is profitable. For any task $t_{i}$, the start time $t_{s}^{i}$ and end time $t_{e}^{i}$ can be calculated via Equations (4) and (5). The completion time of a batch job could be evaluated by the termed of Equation (6). The function pre $\left(t_{i}\right)$ gets all the direct upstream tasks of $t_{i}$. Take Hadoop MapReduce as an example. The number of map tasks can be estimated by data volume of involved input, and their parallelism can be deduced by task number. The reduce tasks is set according to the job configuration and triggered to execute after map tasks' completion. Accordingly, the executive sequence of such a job can be built by coordinator. The constant $\mathrm{D}_{j i}$ depicts the data transmission time from $t_{j}$ to $t_{i}$. If $t_{j}$ and $t_{i}$ are placed in the same worker (i.e., $x_{i}=x_{j}$ ), then $\mathrm{D}_{j i}$ is related with processed data volume and selectivity [Dias de Assunção et al. 2018] of $t_{j}$; otherwise, it is also related the bandwidth between two nodes. The constant $\mathrm{W}_{i}$ describes the working time of task $t_{i}$ with certain calculative operations. Managed in a coordinator, these two constants could be achieved statistically, because the selectivity of common business operation is definite. In highway domain, the descriptive statistics are concluded in our previous work [Ding et al. 2020],

$$
\begin{aligned}
& t_{s}^{i}=\left\{\begin{array}{c}
0 \\
\max \left\{t_{e}^{j}+\mathrm{D}_{j i}: t_{j} \in \operatorname{pre}\left(t_{i}\right)\right\}, \text { otherwise }
\end{array}\right. \\
& t_{e}^{i}=t_{s}^{i}+\mathrm{W}_{i}, \\
& \text { makeSpan }=\max \left\{t_{e}^{i}\right\} .
\end{aligned}
$$

For a stream application, lower communication among workers for an application is favorable. The availability of an application is proved decreasing when involved machines increase through traditional random placement [Ding et al. 2016]. Accordingly, the workers where tasks are placed are expected to be minimized with given resource capacities. As Equations (7) and (8), the number of involved workers is presented.

$$
\begin{aligned}
& Y_{0}(j)= \begin{cases}1, & \text { if } \sum_{i=1}^{\mathrm{T}} Y_{0}(i, j) \geq 1 \\
0, & \text { otherwise }\end{cases} \\
& \text { nodeSize }=\sum_{j=1}^{\mathrm{R}} Y_{0}(j) .
\end{aligned}
$$

With different considerations for batch and stream analytics, the evaluation for an iteration of our placement method can be defined as follows.

Definition 4 (Fitness Function). The fitness function $f$ is a value to evaluate the merits of iteration, and lower is better. It is depicted respectively for the batch and stream analytical application, 
Table 3. Task Placement Algorithm Through Particle Swarm Optimization

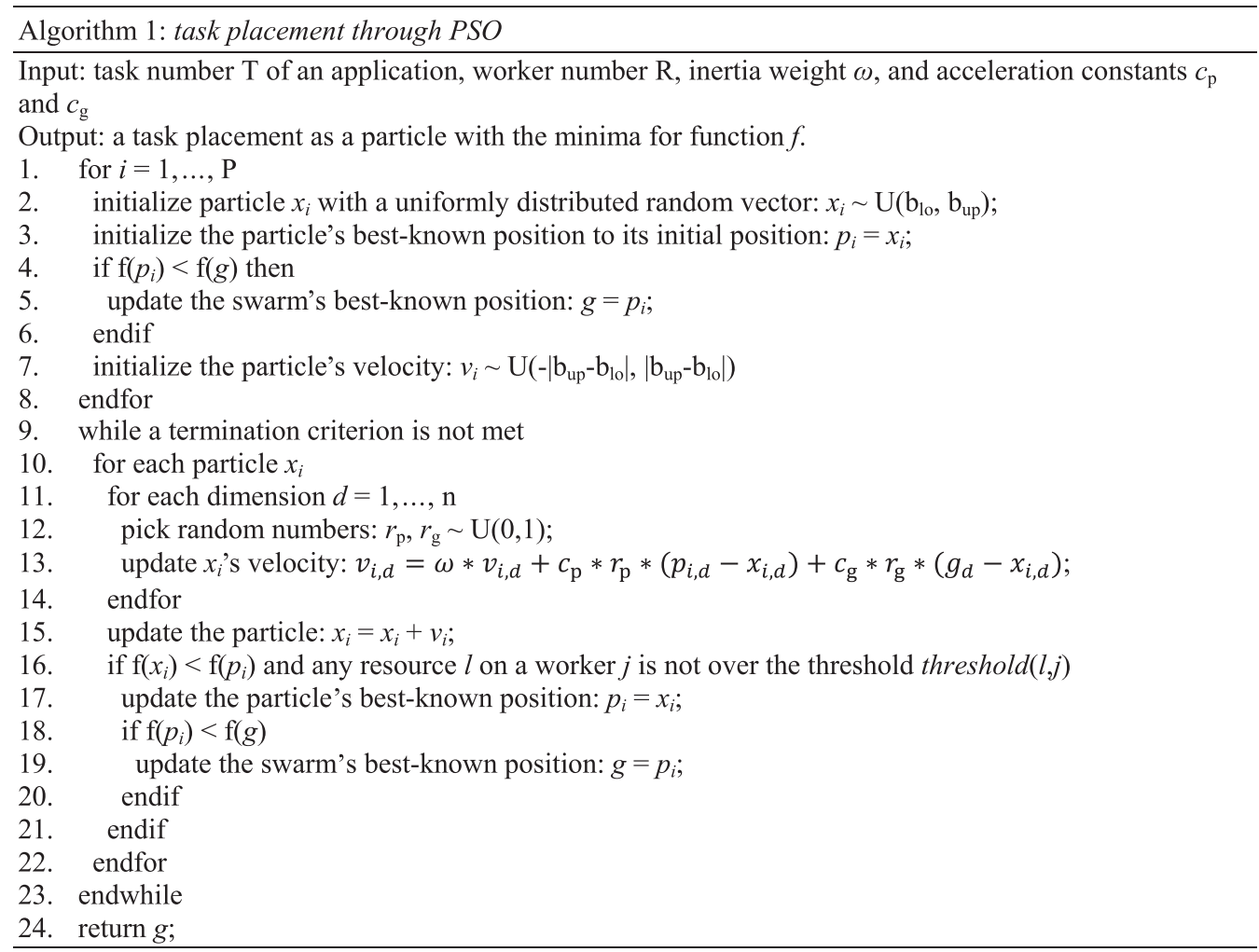

$$
f=\left\{\begin{array}{l}
1 / \text { makeSpan, jobType }=\text { batch } \\
\text { nodeSize, jobType }=\text { stream }
\end{array} .\right.
$$

With this fitness function, the PSO procedure for the task placement of an application can be abstracted as an optimization problem below and then is implemented as the algorithm in Table 3.

$$
\begin{array}{ll}
\operatorname{minimize} & f \\
\text { s.t. } & Y_{l}(j)=\sum_{i=1}^{\mathrm{T}} Y_{0}(i, j) \leq \text { threshold }(l, j), \forall l
\end{array}
$$

Here, the algorithmic parameters, inertia weight $\omega$, and the acceleration constants (i.e., $c_{\mathrm{p}}$ and $c_{\mathrm{g}}$ ) would be tuned and evaluated by cross-validation. In lines $1-8$, with $f(g)=\infty$ initially, the original swarm is initialized where the constant $P$ implies the swarm size. The constants $b_{l o}$ and $b_{\text {up }}$ are the lower and upper boundaries of search-space. The termination criterion in line 9 is the maximum of iterations, or an invariant solution is found. Each particle is updated in lines 11-15. The bestknown position of the particle and the swarm are updated in lines 16-21. Here, the threshold of available resource $l$ on the worker node $j$ in line 16 is designated in coordinator.

In summary, on resource of available workers, this proposed method tries to achieve optimal executive time for a batch analytical application and reduce communication consumption for a stream one. 


\subsection{Adaptive Task Scheduling Against Sudden Workload}

Tasks of applications are continuously supervised by coordinator at runtime, and some of them have to be re-scheduled to additional workers when sudden workloads appear. Such dynamical task allocation is termed as adaptive task scheduling in this article, which is a workload-aware method based on short-term workload prediction. Different with the initial task placement, the adaptive task scheduling only focuses on stream analytical applications. For batch ones with Hadoop MapReduce or Spark, bottleneck tasks would be regarded as a failure through the speculation mechanism and would be automatically redeployed to other worker [Zaharia 2014]. However, in long running stream computing, such a mechanism would directly affect both upstream and downstream ones of a bottleneck task [Ding et al. 2016], and may deteriorate processing latency dramatically. Therefore, the adaptability is not trivial in stream computing, especially under sudden bursting and shrinking workload.

Definition 5 (Workload, Bursting Workload, and Shrinking Workload). For a task $t_{i}$, workload depicts the rate of incoming tuples in a given time slot and is measured by the coordinator. In recent $N$ observations, a workload is termed bursting if the proportion of the predictive values larger than the high mark $m_{h}^{i}$ is more than threshold $H$; a workload is termed shrinking if the proportion of the predictive values smaller than the low mark $m_{l}^{i}$ is more than threshold $L$. By default, the threshold $H=L=50 \%$; for any task $t_{i}$, the high mark $m_{h}^{i}$ is identical to the bandwidth of the worker it deployed on, and the low mark $m_{l}^{i}$ is zero.

Definition 6 (Straggler Task and Spare Task). A straggler task is the one with bursting workload; a spare task is the one with shrinking workload. These two types of tasks are apt to be performance bottleneck and ought to be re-allocated at runtime.

For stream analytics, a straggler is such a task whose processing cannot keep up with its workload and unusually takes long time to complete its computational operations. It is always computing intensive and on a worker with high resource occupancy. Contrarily, a spare task is always deployed on a worker with surplus resources.

According to those definitions, workload prediction is critical to find the bottlenecks. Based on Support-vector Regression (SVR), an effective method is designed in coordinator for task supervision. Due to limited volume and dimensions of input data in a task, it guarantees fast response for prediction and low latency to find a straggler or spare task.

Definition 7 (Workload Encoding). The workload of task $t_{i}$ can be encoded as a vector $w_{i}^{t}=\left(x_{i}^{t}, y_{i}^{t}\right)$ with $2^{*} \theta$ dimensions: $y_{i}^{t}=i n_{i}^{t} ; x_{i}^{t}=\left(i n_{i}^{t-1}, \ldots, i n_{i}^{t-m}, \ldots, i n_{i}^{t-\theta+1}, \sum_{j}\right.$ out $t_{j}^{t}$, $\sum_{j}$ out $_{j}^{t-1}, \ldots, \sum_{j}$ out $_{j}^{t-m}, \ldots, \sum_{j}$ out $\left.t^{t-\theta+1}\right)$. Here, $t$ is the current time slot $t\left(t_{l}, t_{r}\right)$, window length $\left(t_{r}-t_{l}\right)$ is a constant; $t-m$ is the $m^{\text {th }}$ previous time slot, $m \in \mathbb{Z}$, and $\theta$ is a temporal constant. $i n_{i}^{t}$ and out $t_{i}^{t}$, respectively, present the input and output data volume of task $t_{i}$ at time slot $t$. Task $t_{j} \in$ pre $_{\text {direct }}\left(t_{i}\right)$ is a direct upstream one of $t_{i}$ in this analytical application.

The encoded workload vector is composed by two parts. One is the input data volume of task $t_{i}$ in recent $\theta$ time slots, which considers the characteristics of recent time series. The temporal constant $\theta$ is an additional algorithmic parameter. The other part is the output data volume of all its direct upstream tasks in recent $\theta$ time slots. It reflects the characteristics of topological locality, where the input of task $t_{i}$ is dependent on the output of topological upstreams. To limit the size of vector dimension, only the most relevant tasks (i.e., direct upstream ones) are considered here.

Originated from support-vector machine, SVR model depends only on a subset of training data and can tolerate some margin points. With the help of kernel function, low-dimensional data could 
Table 4. Task Workload Prediction Algorithm Through Support-vector Regression

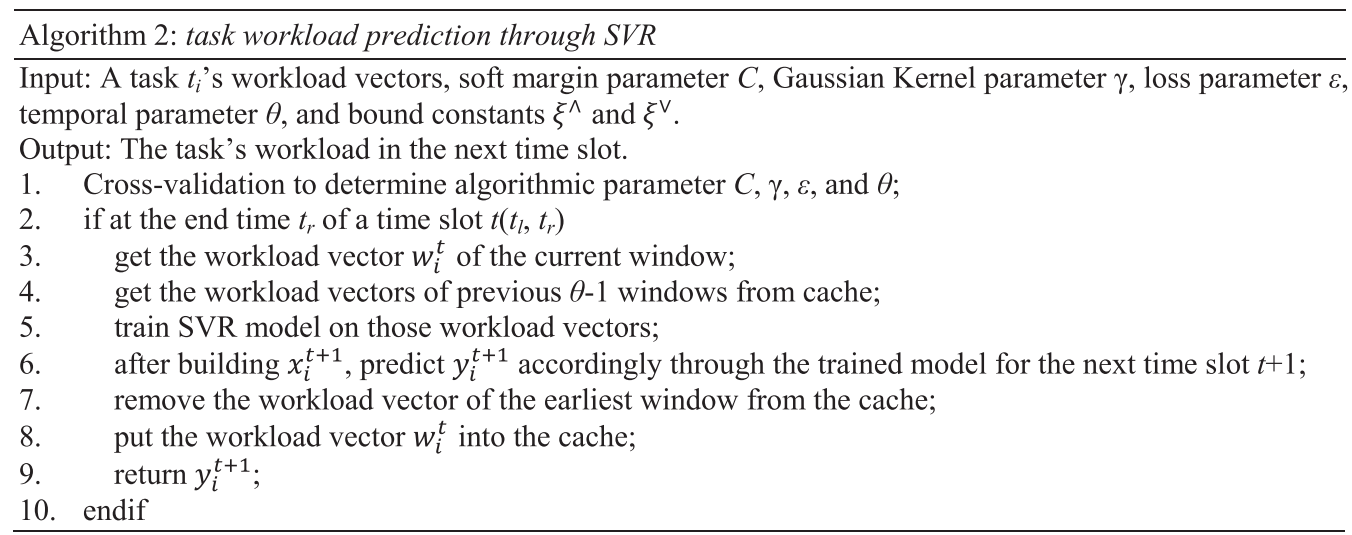

be separable in a high-dimensional space by non-linear transformation. Here, the Gaussian Kernel $K(x, z)=\exp \left(-\gamma *\left\|x-z^{2}\right\|\right)$ is adopted as the kernel function with a parameter $\gamma>0$. The effectiveness of the SVR model depends on algorithmic parameters, including soft margin parameter $C$ for auxiliary slack variables, Gaussian Kernel parameter $\gamma$, loss parameter $\varepsilon$, and vector temporal parameter $\theta$. Generally, the combination of parameter choices should be cross-validated, and the parameters with best fitness are picked manually. With given fitness function, the workload prediction through SVR can be abstracted as an optimization problem as follows:

$$
\begin{array}{ll}
\operatorname{minimize} & \frac{1}{2}\|\omega\|^{2}+C \sum_{i=1}^{\theta}\left(\xi^{\wedge}+\xi^{\vee}\right) \\
\text { s.t. } & y_{i}-\left(\omega * x_{i}+b\right) \leq \varepsilon+\xi^{\wedge} \\
& \left(\omega * x_{i}+b\right)-y_{i} \leq \varepsilon+\xi^{\vee} \\
& \xi^{\wedge}, \xi^{\vee} \geq 0 .
\end{array}
$$

Here, the optimal hyper plane is found to regress workload vectors in non-linear space and would be used to predict the workload in next time slot. In such a hyper plane, $\omega$ is the coefficient of the dimensions and $b$ is the intercept vector. For geometrical margin of tolerance, $\varepsilon$ is the slack variables with the bound $\xi^{\wedge}$ and $\xi^{\vee}$ and $C$ is soft margin parameter. Accordingly, task's workload prediction could be implemented as the algorithm in Table 4 .

The prediction is also a stream computing procedure. The workload vectors of a task are organized by coordinator in time-based tumbling windows, and the ones in previous $\theta-1$ windows have been cached for later usage. That is presented by line 4 and lines 7 and 8 . After training on those vectors in recent $\theta$ windows like line 5 , the model can achieve the predictive workload $y_{i}^{t+1}$ in line 6 for task $t_{i}$ in the next time slot $t+1$.

With this predicted workload, the adaptive task scheduling could be implemented as the procedure in Figure 3. The monitor of coordinator would periodically acquire predictive workloads for all the tasks. After the evaluation, any straggler task would be found and migrated to a target worker. According to Equation (7), such target worker $j$ is an idle one (i.e., $Y_{0}(j)=0$ ) or an active worker (i.e., $Y_{0}(j)=1$ ) with resource not exceeding given thresholds. It is quite different for spare task $t_{s}$ that its migration is done only if all tasks in the same worker with $t_{s}$ are spare ones. Such a worker is regarded as under-loaded and would become an idle one after all its tasks' migration. It makes efficient financial and power cost in IoT environment. 


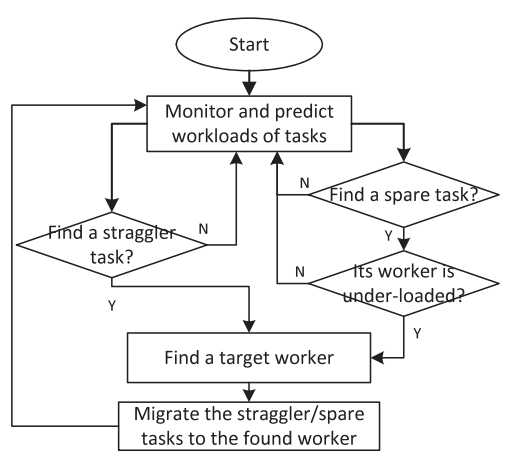

Fig. 3. Adaptive task scheduling.

\section{EVALUATION}

In the practical scene mentioned in Section 3.1, we conduct experiments to quantitatively compare our method with default ways of respective native systems. To evaluate the effects of initial task placement, the time cost, bandwidth utility, and scalability are concerned, and four experiments are realized in Section 5.2. To evaluate the effects of adaptive task scheduling, predictive precision, worker number, bandwidth utility, and scalability are the focus, and three experiments are completed in Section 5.3.

\subsection{Setting}

The experiments involve three layers of hardware. (1) For far-end private Cloud, six of Acer AR580 F2 rack servers via Citrix XenServer 6.2 are utilized, each of which own eight processors (Intel Xeon E5-4607 2.20GHz), 64 GB RAM, and 80 TB storage. (2) For the edge layer, tens of personal computers are used as edge servers, each of which owns four cores CPU (Intel Core i5 4590), 8 GB RAM, and 1 TB storage with CentOS $6.6 \times 86 \_64$ operating system installed. Three levels (i.e., toll station, sectional center, and regional center) of edges are composed in a tree structure as shown in Figure 1. (3) For front-end devices, the sensors are emulated through our dedicated data generator [Ding et al. 2014; Ding et al. 2016], in which concurrency and velocity could be configured by userdefined scripts and configurations. Toll data would be replayed or simulated by this data generator with the seed data generated in April 2017 from the real sensors, where about 45 million records exist. By default, simulated data are generated as the rate of 1 record per $3 \mathrm{~s}$ from a toll station, which is just the statistical mean value of the ground truth.

Our framework with dependent software is configured in the edge layer. (1) In each of the three levels, only one range is organized among edge servers to focus on the key effects. ZeroMQ 2.1.4 is employed as a lightweight message service between different levels' communication. (2) In each range, one edge server is used as coordinator and the others are workers. A tailored Hadoop 2.6.0 and Storm 1.1.0 are configured as a federal combination on those servers. For massive historical data such as toll data, HBase 1.6.0 and HDFS 2.6.0 are employed on TB-level storage; for basic data such as station profile, MySQL 5.6.17 is adopted. In addition, auxiliary distributed service like Kafka 1.0 and ZooKeeper 3.4.5 are installed. (3) In a coordinator, master services like resource manager, application master, and nimbus run as daemons. An analytical application through MapReduce or Storm could be submitted to any available coordinator. (4) In a worker, working services such as node manager and supervisor are deployed as respective daemons.

The analytical applications of traffic volume in highway domain are used for illustration, which counts vehicles passing a spatial location in given time slots. In fact, traffic volume can be depicted 
(a)

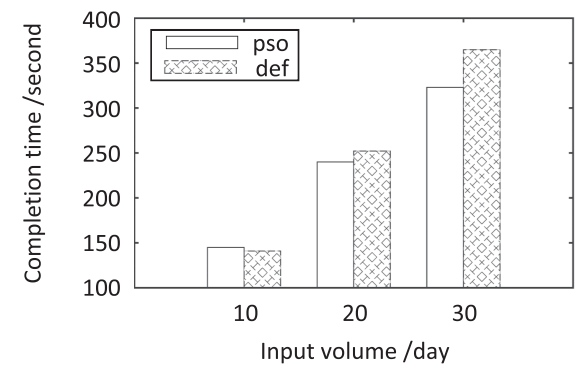

(b)

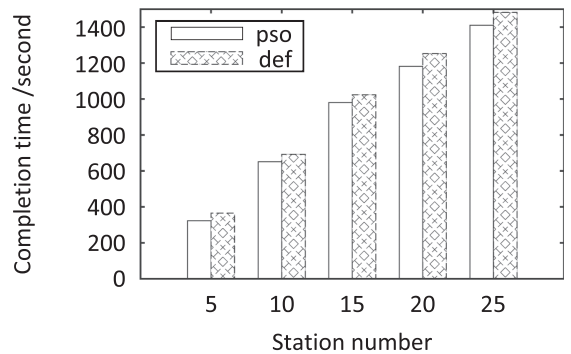

Fig. 4. Average completion time of batch analytics.

in two computing models from technical perspective: The batch model on massive historical data is implemented by Hadoop [Ding et al. 2020] and the stream model on continuous real-time data is realized by Storm through time-based tumbling window [Ding et al. 2020]. Encapsulating existing jobs, some applications would run in our framework. Three key parameters exist here [Ding et al. 2020]. (1) Temporal parameter $t$ is the granularity of time slot, such as 5 minutes, 15 minutes, 30 minutes, 1 day, or one month. By default, $t=5$ minutes. (2) Spatial parameter $s$ shows the granularity of concerned space, such as station, section, region, or province. For example, "STATION" means the analytics would achieve the results on each toll station; while "PROVINCE" would get results of provincial road network as a whole. By default, $s=$ STATION. (3) Moving object parameter $o$ is the concerned type of specific vehicles. For example, some applications only focus on "MTC" vehicles (charged by Manual Toll Collection system), some on ones with "LOCAL" license plate of province, and other may on the type of "FREIGHT-CARRIAGE." By default, $o=$ ALL.

\subsection{Task Placement and Its Effects}

Our method (abbreviated as pso) and default way (abbreviated as def) [Anderson 2013; Rao and Reddy 2012] in respective native systems are compared in their time cost, bandwidth utility, and scalability, where analytical applications of both batch and stream type are submitted. Four experiments are designed for two models with or without edge resource.

Experiment 1. In the private Cloud, a batch application 5 minutes traffic volume of ETC vehicles at station defined as Table 2 is submitted to our framework without offload support; the inclusive MapReduce job is also submitted to native Hadoop as a counterpart. Set parameters are $t=$ 5 minutes, $s=$ STATION, and $o=$ ETC. From 30 days' data of April 2017, the input volume would be added progressively in each test. The completion times for the batch analytics of each test are counted.

The results are presented in Figure 4(a). In either method, the batch analytics would be submitted to our private Cloud for traffic volumes at all the toll stations in that province. When input involves the data of more days, the completion time in either way increases, because more time is required to scan and process larger data. Meanwhile, on the data of the same volume, that time in our method trends to be shorter than that of the counterpart with a wider difference. For the data of 30 days, such difference is about 1 minute. The time reduction comes from resource utility optimization during task placement, in which the solution with the shortest make span is prone to be found. Larger input implies more map tasks for placement, and wider time difference would be between these two methods. While through the default way in native Hadoop, tasks are placed to workers as first-in-first-out (FIFO) policy as long as sufficient resource is available. It cannot fully consider the characteristics of batch computing with resource utilities. 
Table 5. Resource Utilization of Stream Analytics in Cloud

\begin{tabular}{ccccc}
\hline Concurrency & \#-pso & $\%$-pso & \#-def & $\%$-def \\
\hline 50 & 1 & 72 & 3 & 23 \\
100 & 2 & 55 & 3 & 35 \\
200 & 3 & 88 & 3 & 81 \\
300 & 3 & 92 & 3 & 95 \\
400 & 4 & 93 & 3 & 100 \\
500 & 5 & 92 & 3 & - \\
\hline
\end{tabular}

Experiment 2. Another batch application 5 minutes traffic volume of ETC vehicles at section is submitted to a coordinator of our framework with offload support; the inclusive MapReduce job is also submitted to native system Hadoop in our private Cloud as a counterpart. Set parameters are $t=5$ minutes, $s=$ SECTION, and $o=$ ETC. The data of 30 days in April 2017 is imported as input, and the station number would be added progressively in each test. The completion times for the batch analytics of each test are counted.

The results can be found in Figure 4(b). In the hierarchy of edge servers as Figure 1, a region contains several sections, and a section contains multiple toll stations. The addition operation makes traffic volume of a section the sum of the values of toll stations belonging to the very section and also makes the traffic volume of a region the sum of the value of toll stations belonging to the section in the very region. Here, the coordinator that application submitted to is the one in a regional edge server covering given sections. With offload support, the batch application could be migrated to the lower-level workers and summarize the results from them. During time elapses, more toll stations would be introduced. Due to the same reason of Experiment 1, the completion time of either method would increase when more data are generated from more stations. In the same station number, that time in our method trends to shorter than that of the counterpart, because faster analytical response is brought through our task placement method in edge servers close to the data. When station number is larger than 15 , the difference between two methods is almost a constant about 1 minute. It is because that the incremental communication among workers cancels the time reduction from our task placement method.

Experiment 3. In the private Cloud, a stream application 5 minutes traffic volume of ETC vehicles at station is submitted to our framework without offload support; the inclusive topology is also submitted to native Storm as a counterpart. The differences of application definition from that of Table 2 are jobType as stream and jobPackage as another corresponding JAR file. Set parameters are $t=5$ minutes, $s=\mathrm{STATION}$, and $o=\mathrm{ETC}$. The input is simulated stream in which the rate is kept as 1,000 records per second per connection, and different concurrency is configured in each test. The involved workers for the stream analytics of each test are counted after running smoothly.

The results are illustrated in Table 5, where \# means the number of involved workers, \% implies the average bandwidth usage of those workers, and "-" notates unknown due to task's crash. Under the same data rate, the concurrency (i.e., the number of toll stations here) has a 10-fold increase progressively, and we find an auto-scaling guarantee in our method. (1) In native Storm, worker number is a constant, because the workers cannot join in or drop from cluster at runtime unless external intervention. When concurrency grows, the average bandwidth increases due to larger data involved. If it is reaching the resource limitation, then processing is unable to keep up with input rate and would lead block until crash. (2) In our framework, the worker number would be as small as possible to reduce communication among dynamically organized worker and keeps pace with 
Table 6. Resource Utility of Stream Analytics with Edges

\begin{tabular}{ccccc}
\hline Concurrency & \#-pso & \%-pso & \#-def & $\%$-def \\
\hline 50 & 1 & 72 & 3 & 28 \\
100 & 2 & 55 & 3 & 32 \\
200 & 3 & 88 & 3 & 79 \\
300 & 5 & 68 & 3 & 98 \\
400 & 5 & 89 & 3 & - \\
500 & 5 & 92 & 3 & - \\
\hline
\end{tabular}

concurrency accordingly. When the concurrency is larger than 300 , the bandwidth usage is almost close to the restriction $95 \%$ for user-defined QoS, whereas through the default method in native Storm, a task would be placed to a random worker as long as enough slots in that worker are available. It cannot fully consider a key factor for the stream model like communication latency. Therefore, in our private Cloud, auto-scaling with lower communication cost makes our method better.

Experiment 4. Another stream application, 5 minutes traffic volume of ETC vehicles at section, is submitted to a coordinator of our framework with offload support; the inclusive topology is also submitted to native Storm in the private Cloud as a counterpart. Set parameters $t=5$ minutes, $s=$ SECTION, and $o=$ ETC. The input is simulated stream in which the rate is kept as 1,000 records per second per connection and different concurrency is configured in each test. The involved workers for the stream analytics of each test are counted after running smoothly.

The results are illustrated in Table 6 with the same notations as Table 5. Two settings here are different from those of Experiment 3. First, the traffic volume is for sections instead of stations. In the scene of data here, any toll station belongs to a definite section of the five ones. Second, offload support is enabled to employ edge resource. The effects in native Storm appear similar as that of Experiment 3. In our framework, auto-scaling is also guaranteed due to the addition operation of traffic volume analytics. In our method, when additional edge worker of is required, the ones near the data would be used. When concurrency is larger than 300, tasks of the application begin to offload to edge workers in sectional center, which dramatically reduces the time than the counterpart gathering all the data in central Cloud. Therefore, besides the auto-scaling feature like Experiment 3, our method can use more edge resource closing to the data to improve analytical performance accordingly.

\subsection{Workload Prediction and Task Scheduling}

Workload prediction is part of our adaptive task scheduling method, and its precision is evaluated in one experiment here. It is done on data of certain workdays and holidays that appear distinct trends in highway. Two common metrics are employed for evaluation. One is mean absolute percentage error (MAPE) as Equation (10); the other is median absolute percentage error (MDAPE) as Equation (11). Here, at time $t$ for a task, $x_{t}$ is the factual workload and $x_{t}^{\prime}$ is its predictive value achieved at time $t-1$,

$$
\begin{gathered}
\text { MAPE }=\frac{1}{N} \sum_{1}^{N} \frac{\left|x_{t}-x_{t}^{\prime}\right|}{x_{t}} * 100 \%, \quad t=1,2 \ldots \in \mathbb{Z}, \\
\text { MDAPE }=\operatorname{median}\left(\frac{\left|x_{1}-x_{1}^{\prime}\right|}{x_{1}} * 100 \%, \ldots, \frac{\left|x_{i}-x_{i}^{\prime}\right|}{x_{i}} * 100 \%, \ldots\right), i=1,2 \ldots \in \mathbb{Z} .
\end{gathered}
$$

Experiment 5. The data for a workday (April 20, 2017) and those of a holiday (February 11, 2017; the Lantern Festival on a Saturday) are replayed respectively as stream by our data generator. The 
Table 7. Workload Prediction Effects

\begin{tabular}{ccccc}
\hline Item & w-svr & h-svr & w-arima & h-arima \\
\hline MAPE(\%) & 5.43 & 27.11 & 11.94 & 23.71 \\
MDAPE(\%) & 4.22 & 5.37 & 5.68 & 6.81 \\
Latencies(ms) & 1875 & 1863 & 3560 & 2488 \\
\hline
\end{tabular}

(a)

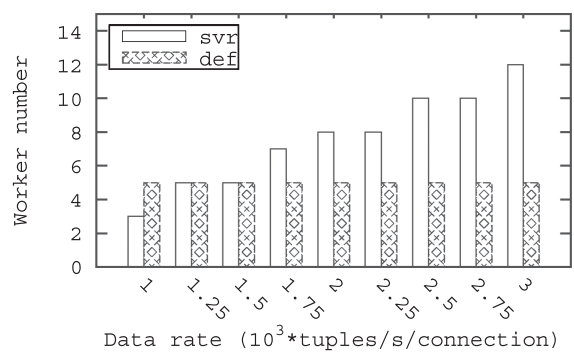

(b)

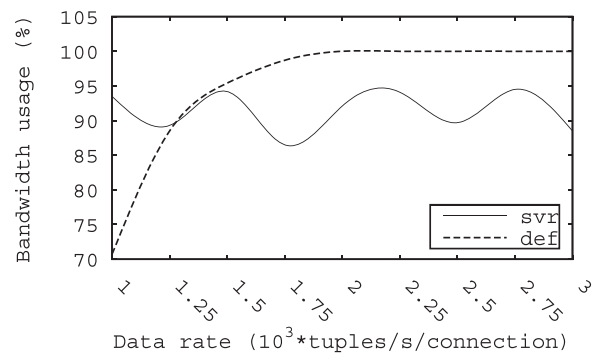

Fig. 5. Adaptive task scheduling under bursting workload.

same application in Experiment 3 is submitted to our framework in the private Cloud without offload support. In coordinator, both time-series model ARIMA (abbreviated as arima) [Ding and Zhao 2018] and our method (abbreviated as svr) are implemented to predict the workload every 5 minutes (i.e., identical with parameter $t$ of the application). After the application runs smoothly, outputs and latencies of the predictions are counted, and the average of two metrics MAPE and MDAPE for all the stations can be computed with the ground truth.

The result is shown in Table 7, where $w$ and $h$ mean weekday and holiday, respectively. Our method holds a little higher accuracy with much lower latency than the counterpart. (1) In both methods, any of the two metrics is acceptable with different precision in workday and holiday. Comparing MAPE in Table 7, both methods fit better on a workday, because the traffic pattern on a holiday is not so obvious. Moreover, in our method little difference between two metrics on workday implies more steady predictive effects. (2) Our method presents better effects. On the one hand, it shows relatively lower errors especially in MDAPE, because not only the time but also the spatial factors (i.e., upstream workload) are modelled during the prediction. On the other hand, our method has distinct advantages in predictive latency. The latencies on workday are almost the same with that of holiday due to non-linear separation. Our method cuts down more than $25 \%$ about required time, because fewer coefficients to train make the prediction faster.

Then, our method (svr) and default way (def) [Anderson 2013] in native Storm are compared in their worker number, bandwidth utility, and scalability, where the stream applications are observed. Two experiments are designed under bursting and shrinking workload.

Experiment 6. In the private Cloud as Experiment 3, the same application is submitted to our framework without offload support; the inclusive topology is also submitted to native Storm with five workers. The window length of stream analytics is 5 minutes. In our data generator, a simulated data stream is employed: set concurrency as 300; configure default data rate as 1,000 records per second per connection; increase data rate from 1,000 to 3,000 records per second per connection progressively in 30 selected toll stations. After the stream analytics having run smoothly, the involved workers are counted.

Figure 5 shows the worker number and the average bandwidth usage of workers. On the one hand, as shown in Figure 5(a), our method proves auto-scaling when the data rate of selected 
(a)

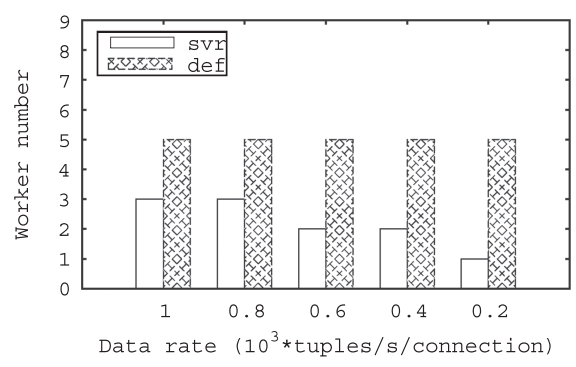

(b)

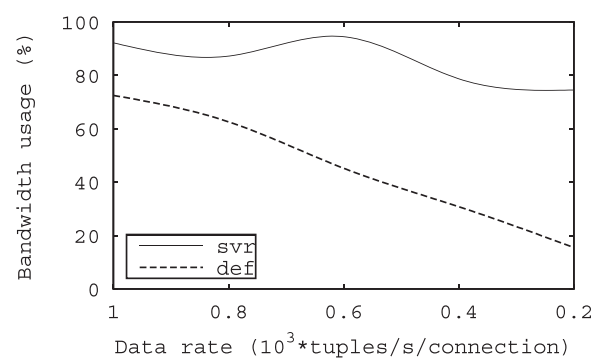

Fig. 6. Adaptive task scheduling under shrinking workload.

stations grows. The incremental data rate leads to bursting workload and makes some tasks straggler ones. Through the adaptive task scheduling in our framework, the worker number would be invariant if such straggler tasks are migrated to another worker with low bandwidth occupancy; otherwise, worker number would increase if straggler tasks are deployed to a new idle worker. But in native Storm, the worker cannot adjust according to such dynamic workload. On the other hand, as shown in Figure 5(b), the bandwidth utility of involved workers is relatively steadier (about 85-95\%) than the counterpart, because our method can minimize worker number as much as possible. Under a given workload, the smaller the number of the worker, the higher bandwidth usage would be. It also explains why in a native system the bandwidth usage increases sharply to reach resource limitation and the straggler tasks are prone to crash due to head-of-line blocking. Therefore, our method can adapt to bursting workload for straggler tasks flexibly.

Experiment 7. In the private Cloud as Experiment 3, the same application is submitted to our framework without offload support; the inclusive topology is also submitted to native Storm with five workers. The window length of stream analytics is 5 minutes. In our data generator, simulated data stream is employed: set concurrency as 300; configure default data rate as 1,000 records per second per connection; decrease data rate from 1,000 to 200 records per second per connection progressively in 30 selected toll stations. After the stream analytics having run smoothly, the involved workers are counted.

The result is presented in Figure 6, which uses the same notations as Figure 5. The auto-scaling capacity of our method is proved again in Figure 6(a) when data rate of selected stations decreases. Such shrinking workload makes some tasks spare ones. Through our adaptive task scheduling, the spare tasks on under-loaded worker would be migrated to other workers with low bandwidth occupancy. The worker number declines after the tasks' migration and the under-loaded worker becomes idle. Moreover, as shown in Figure 6(b), the steadily high utility (about $80-95 \%$ ) of the average bandwidth is guaranteed, because surplus under-loaded workers are not used by the application after spare tasks' migration. However, workers in native Storm cannot adjust according to such dynamic workload, and wasted resource leads to lower bandwidth utilities. Therefore, under shrinking workload, our method can hold resource utilities accordingly and would save power or financial cost due to economic resource.

\section{CONCLUSION}

For urban IoT applications, hybrid analytical models of batch and stream computing are supported in our unified framework, where resources of Cloud and Edge are collaborated effectively. Two task allocation methods are presented accordingly to optimize resource utilities in two different conditions. When an application is submitted, the task placement method based on particle 
swarm optimization can distinctly reduce the completion time for batch tasks and maximize the bandwidth utilization of existing workers for stream tasks; when sudden bursting or shrinking workloads appears, the adaptive task scheduling method on predictive workloads would guarantee steady and high bandwidth utility for the stream tasks. Evaluated in a practical scene of highway domain, our work shows efficient utilization improvement with the auto-scaling guarantee on both real-world and simulated data.

In future, we will focus on fine-grained workload prediction with more runtime features, which would improve the adaptability of our task scheduling method.

\section{REFERENCES}

T. Akidau, A. Balikov, K. Bekiroglu, S. Chernyak, J. Haberman, R. Lax, S. Mcveety, D. Mills, P. Nordstrom, and S. Whittle. 2013. MillWheel: Fault-tolerant stream processing at internet scale. In Proceedings of the 39th International Conference on Very Large Data Bases (VLDB'13). 734-745.

Q. Anderson. 2013. Storm Real-time Processing Cookbook. Packt Publishing Ltd.

L. Aniello, R. Baldoni, and L. Querzoni. 2013. Adaptive online scheduling in storm. In Proceedings of the Proceedings of the 7th ACM international Conference on Distributed Event-based Systems. ACM, 207-218.

O. Boykin, S. Ritchie, I. O'Connell, and J. Lin. 2014. Summingbird: A framework for integrating batch and online MapReduce computations. Proc. VLDB Endow. 7, 13 (2014), 1441-1451.

Y. Cao and H. Wang. 2015. The key technologies of real-time processing large scale microblog data stream. In Cloud Computing and Big Data, W. Qiang, X. Zheng, and C.-H. Hsu (Eds.). Springer International Publishing, Cham, 295-306.

P. Carbone, S. Ewen, S. Haridi, A. Katsifodimos, V. Markl, and K. Tzoumas. 2015. Apache Flink: Stream and batch processing in a single engine. IEEE Bull. IEEE Comput. Soc. Techn. Commit. Data Eng. 36, 4 (2015), 28-38.

G. J. Chen, J. L. Wiener, S. Iyer, A. Jaiswal, R. Lei, N. Simha, W. Wang, K. Wilfong, T. Williamson, and S. Yilmaz. 2016. Realtime data processing at facebook. In Proceedings of the 2016 International Conference on Management of Data. ACM, 1087-1098.

H. Cho, H. Shiokawa, and H. Kitagawa. 2016. Jsflow: Integration of massive streams and batches via json-based dataflow algebra. In Proceedings of the 19th International Conference on Network-Based Information Systems (NBiS'16). IEEE, 188195.

O. M. Dias De Assun, A. Da Silva Veith, and R. Buyya. 2018. Distributed data stream processing and edge computing: A survey on resource elasticity and future directions. f. Netw. Comput. Appl. 103 (2018), 1-17.

W. Ding, Y. Han, J. Wang, and Z. Zhao. 2014. Feature-based high-availability mechanism for quantile tasks in real-time data stream processing. Softw.: Pract. Exper. 44, 7 (2014), 855-871.

W. Ding, X. Wang, and Z. Zhao. 2020. CO-STAR: A collaborative prediction service for short-term trends on continuous spatio-temporal data. Fut. Gener. Comput. Syst. 102 (2020), 481-493.

W. Ding, S. Zhang, and Z. Zhao. 2017. A collaborative calculation on real-time stream in smart cities. Simul. Model. Pract. Theory 73 (2017), 72-82.

W. Ding and Z. Zhao. 2018. DS-Harmonizer: A harmonization service on spatio-temporal data stream in edge computing environment. Wireless Commun. Mobile Comput. 2018 (2018), 12.

W. Ding, Z. Zhao, and Y. Han. 2016. An adaptive replica mechanism for real-time stream processing. In Proceedings of the 2016 International IEEE Conferences on Ubiquitous Intelligence \& Computing, Advanced and Trusted Computing, Scalable Computing and Communications, Cloud and Big Data Computing, Internet of People, and Smart World Congress (UIC/ATC/ScalCom/CBDCom/IoP/SmartWorld'16). IEEE, 449-455.

W. Ding, Z. Zhao, and Y. Han. 2016. A framework to improve the availability of stream computing. In Proceedings of the 23rd IEEE International Conference on Web Services (ICWS'16). IEEE, 594-601.

W. Ding, J. Zou, and Z. Zhao. 2020. A multidimensional service template for data analysis in highway domain. International Journal of Internet Manufacturing and Services 4, 4 (2020), 290-306.

I. Farris, L. Militano, M. Nitti, L. Atzori, and A. Iera. 2015. Federated edge-assisted mobile clouds for service provisioning in heterogeneous IoT environments. In Proceedings of the 2015 IEEE 2nd World Forum on Internet of Things (WF-IoT'15). 591-596.

A. J. Ferrer, J. M. Marques, and J. Jorba. 2019. Towards the decentralised Cloud: Survey on approaches and challenges for mobile, ad hoc, and edge computing. ACM Comput. Surv. 51 (2019), 1-36.

B. I. Ismail, E. M. Goortani, M. B. A. Karim, W. M. Tat, S. Setapa, J. Y. Luke, and O. H. Hoe. 2015. Evaluation of Docker as Edge computing platform. In Proceedings of the IEEE Conference on Open Systems (ICOS'15). 130-135.

P. P. Jayaraman, J. B. Gomes, H. Nguyen, Z. S. Abdallah, S. Krishnaswamy, and A. Zaslavsky. 2015. Scalable energy-efficient distributed data analytics for crowdsensing applications in mobile environments. IEEE Trans. Comput. Soc. Syst. 2 (2015), $109-123$. 
T. Kolajo, O. Daramola, and A. Adebiyi. 2019. Big data stream analysis: A systematic literature review. f. Big Data 6 (2019), 47.

J. H. Lampton. 2015. Pig squeal: Bridging batch and stream processing using incremental updates. In Computer Science. University of Maryland.

B. Li. 2015. A platform for scalable low-latency analytics using mapreduce. In Computer Science. University of Massachusetts-Amherst, 378.

T. Locher and A. C. Sima. 2016. Cyclone: Unified stream and batch processing. In Proceedings of the 45th International Conference on Parallel Processing Workshops (ICPPW'16). IEEE, 220-229.

B. Lohrmann, P. Janacik, and O. Kao. 2015. Elastic stream processing with latency guarantees. In Proceedings of the International Conference on Distributed Computing Systems (ICDCS'15).

P. G. Lopez, A. Montresor, D. Epema, A. Datta, T. Higashino, A. Iamnitchi, M. Barcellos, P. Felber, and E. Riviere. 2015. Edge-centric computing: Vision and challenges. SIGCOMM Comput. Commun. Rev. 45 (2015), 37-42.

Y. Lv, Y. Duan, W. Kang, Z. Li, and F. Y. Wang. 2015. Traffic flow prediction with big data: A deep learning approach. IEEE Trans. Intell. Transport. Syst. 16 (2015), 865-873.

P. Mach and Z. Becvar. 2017. Mobile edge computing: A survey on architecture and computation offloading. IEEE Commun. Surv. Tutor. 19 (2017), 1628-1656.

A. Machen, S. Wang, K. K. Leung, B. J. Ko, and T. Salonidis. 2016. Migrating running applications across mobile edge clouds: poster. In Proceedings of the 22nd Annual International Conference on Mobile Computing and Networking. ACM, New York City, NY, 435-436.

R. Mahmud, R. Kotagiri, and R. Buyya. 2018. Fog Computing: A taxonomy, survey and future directions. In Internet of Everything: Algorithms, Methodologies, Technologies and Perspectives, B. Di Martino, K.-C. Li, L. T. Yang, and A. Esposito (Eds.). Springer, Singapore, 103-130.

M. Masdari, F. Salehi, M. Jalali, and M. Bidaki. 2017. A survey of pso-based scheduling algorithms in cloud computing. F. Netw. Syst. Manag. 25 (2017), 122-158.

M. Masdari, S. Valikardan, Z. Shahi, and S. I. Azar. 2016. Towards workflow scheduling in cloud computing: A comprehensive analysis. 7. Netw. Comput. Appl. 66 (2016), 64-82.

M. Mukherjee, L. Shu, and D. Wang. 2018. Survey of fog computing: Fundamental, network applications, and research challenges. IEEE Commun. Surv. Tutor. 20 (2018), 1826-1857.

M. S. H. Nazmudeen, A. T. Wan, and S. M. Buhari. 2016. Improved throughput for Power Line Communication (PLC) for smart meters using fog computing based data aggregation approach. In Proceedings of the 2016 IEEE International Smart Cities Conference (ISC2'16). 1-4.

C. Pahl, S. Helmer, L. Miori, J. Sanin, and B. Lee. 2016. A Container-Based Edge Cloud PaaS Architecture Based on Raspberry Pi Clusters. In Proceedings of the 2016 IEEE 4th International Conference on Future Internet of Things and Cloud Workshops (FiCloudW'16). 117-124.

B. Peng, M. Hosseini, Z. Hong, R. Farivar, and R. Campbell. 2015. R-Storm: Resource-aware scheduling in storm. In Proceedings of the 16th Annual Middleware Conference. ACM, 149-161.

C. Perera, Y. Qin, J. C. Estrella, S. Reiff-Marganiec, and A. V. Vasilakos. 2017. Fog computing for sustainable smart cities: A survey. ACM Comput. Surv. 50 (2017), 1-43.

G. Premsankar, M. D. Francesco, and T. Taleb. 2018. Edge computing for the Internet of Things: A case study. IEEE IoT f. 5 (2018), 1275-1284.

Z. Qian, Y. He, C. Su, Z. Wu, H. Zhu, T. Zhang, L. Zhou, Y. Yu, and Z. Zhang. 2013. TimeStream: Reliable stream computation in the cloud. In Proceedings of the European Conference on Computer Systems (Eurosys'13).

B. T. Rao and L. Reddy. 2012. Survey on improved scheduling in Hadoop MapReduce in cloud environments. arXiv preprint arXiv:1207.0780.

L. Salmon, C. Ray, and C. Claramunt. 2015. A hybrid approach combining real-time and archived data for mobility analysis. In Proceedings of the 6th ACM SIGSPATIAL International Workshop on GeoStreaming. ACM, 43-48.

K.-U. Sattler and F. Beier. 2013. Towards elastic stream processing: Patterns and infrastructure. In Proceedings of the 1st International Workshop on Big Dynamic Distributed Data (BD3'13). Citeseer, 49.

E. Saurez, K. Hong, D. Lillethun, U. Ramachandran, and B. Ottenwalder. 2016. Incremental deployment and migration of geo-distributed situation awareness applications in the fog. In Proceedings of the 10th ACM International Conference on Distributed and Event-based Systems. ACM, 258-269.

W. Shi, J. Cao, Q. Zhang, Y. Li, and L. Xu. 2016. Edge Computing: Vision and Challenges. IEEE IoT f. 3 (2016), 637-646.

R. Tolosana-Calasanz, J. Á. Ba Ares, C. Pham, and O. F. Rana. 2016. Resource management for bursty streams on multitenancy cloud environments. Fut. Gener. Comput. Syst. 55 (2016), 444-459.

S. Yangui, P. Ravindran, O. Bibani, R. H. Glitho, N. B. Hadj-Alouane, M. J. Morrow, and P. A. Polakos. 2016. A platform as-a-service for hybrid cloud/fog environments. In Proceedings of the 2016 IEEE International Symposium on Local and Metropolitan Area Networks (LANMAN'16), 1-7. 
W. Yu, F. Liang, X. He, W. G. Hatcher, C. Lu, J. Lin, and X. Yang. 2018. A survey on the edge computing for the Internet of Things. IEEE Access 6 (2018), 6900-6919.

M. Zaharia. 2014. An architecture for fast and general data processing on large clusters. Doctor of Philosophy in Computer Science, Electrical Engineering and Computer Sciences, University of California at Berkeley.

W. Zhang, H. Lv, L. Xu, Y. Liu, X. Liu, Q. Lu, Z. Li, and J. Zhou. 2017. An Online-Offline Combined Big Data Mining Platform. In Proceedings of the IEEE 14th International Conference on Dependable, Autonomic and Secure Computing, 14th International Conference on Pervasive Intelligence and Computing, 2nd International Conference on Big Data Intelligence and Computing and Cyber Science and Technology Congress (DASC/PiCom/DataCom/CyberSciTech'17). 1220-1225.

Z. Zhao, W. Ding, J. Wang, and Y. Han. 2015. A hybrid processing system for large-scale traffic sensor data. IEEE Access 3 (2015), 2341-2351.

J. Y. Zhu, J. Xu, and V. O. K. Li. 2016. A Four-Layer Architecture for Online and Historical Big Data Analytics. In Proceedings of the IEEE 14th International Conference on Dependable, Autonomic and Secure Computing, 14th International Conference on Pervasive Intelligence and Computing, 2nd International Conference on Big Data Intelligence and Computing and Cyber Science and Technology Congress (DASC/PiCom/DataCom/CyberSciTech 2016). IEEE, 634-639.

Received June 2019; revised October 2019; accepted November 2019 